\title{
SLANT TRANSFORM WATERMARKING FOR TEXTURED IMAGES
}

\author{
Anthony T. S. Ho, Xunzhan Zhu, Yong Liang Guan and Pina Marziliano \\ Nanyang Technological University \\ School of Electrical and Electronic Engineering \\ Singapore, 639798
}

\begin{abstract}
In this paper, we propose a digital watermarking algorithm based on the Slant transform for the copyright protection of complex textured images. A lot of images in practical applications, such as remote sensing and medical archiving, contain complex textures. With more AC energies concentrating in the high frequency components, images with complex textures usually suffer more from common image processing operations than smooth images do. Therefore, watermarking robustness is more an issue for textured images though it is commonly recognized that highly textured images provide more capacity for data hiding without introducing too much degradation to the visual quality of original images. Experimental results showed that our Slant transform watermarking provides significant advantage for watermark insertion and retrieval for images with complex textures such as satellite images. An analytical comparative study on the performance of the Slant transform adapting our earlier watermarking schemes for fast Hadamard transform will be performed based on its robustness against various Stirmark and Checkmark attacks.
\end{abstract}

\section{INTRODUCTION}

Copyright protection and authentication of digital multimedia contents is increasingly becoming more and more important in parallel with the tremendous development of the Internet. To counter this growing information security problem of illegal distribution and counterfeiting, much research effort has been invested in digital watermarking in the past few years. A lot of images in practical applications, such as remote sensing and medical archiving, contain complex textures. Although it is commonly recognized that highly textured images provide more capacity for data hiding without introducing too much degradation to the visual quality of original images, watermarking robustness is more an issue for these images for the reason that usually textured images suffer more from common image processing operations than smooth images.

In this paper, we propose an improved digital watermarking algorithm based on the Slant transform for the copy- right protection of textured images. The Slant transform has been applied to many image processing applications, such as transform coding and image restoration $[1,2,3]$ along with many fast algorithms have been proposed for it [4]. The Slant transform has many similar properties to the Walsh-Hadamard transform. Designed to match basis vectors to areas of constant luminance slope, the Slant transform is good for compact energy in "smooth" images. However, in terms of watermarking, it works better for textured images, for the energy spread becomes a significant advantage in watermarking and offers a good spread of middle to higher frequencies with significant energies for robust information hiding. This has brought us to consider protecting the copyright of textured images using the Slant transform watermarking.

The proposed algorithm will be evaluated based on its robustness against various Stirmark [5] and Checkmark [6] attacks using test images with different textures. The performance comparison results of the Slant transform for complex textured image watermarking against Hadamard transform will also be presented. The rest of this paper is organized as follows. In Section 2, the Slant transform is briefly reviewed. Then, our proposed watermarking method is elaborated in Section 3. Simulation results are presented in Section 4, these include comparison of the Slant transform scheme to the Hadamard transform scheme in [7, 8]. Finally, the conclusions are given in Section 5.

\section{THE SLANT TRANSFORM}

Let $\mathbf{U}$ be the original image of size $N \times N$, its 2-D Slant transform is given by

$$
\mathbf{V}=\mathbf{S}_{N} \mathbf{U} \mathbf{S}_{N}^{T}
$$

where $\mathbf{S}_{N}$ is the $N \times N$ unitary Slant matrix. The inverse transformation to recover $\mathbf{U}$ from the transform components matrix $\mathbf{V}$ is given by

$$
\mathbf{U}=\mathbf{S}_{N}^{T} \mathbf{V} \mathbf{S}_{N} .
$$

The Slant transform is a member of the orthogonal transforms. It has a constant function for the first row, and has a 


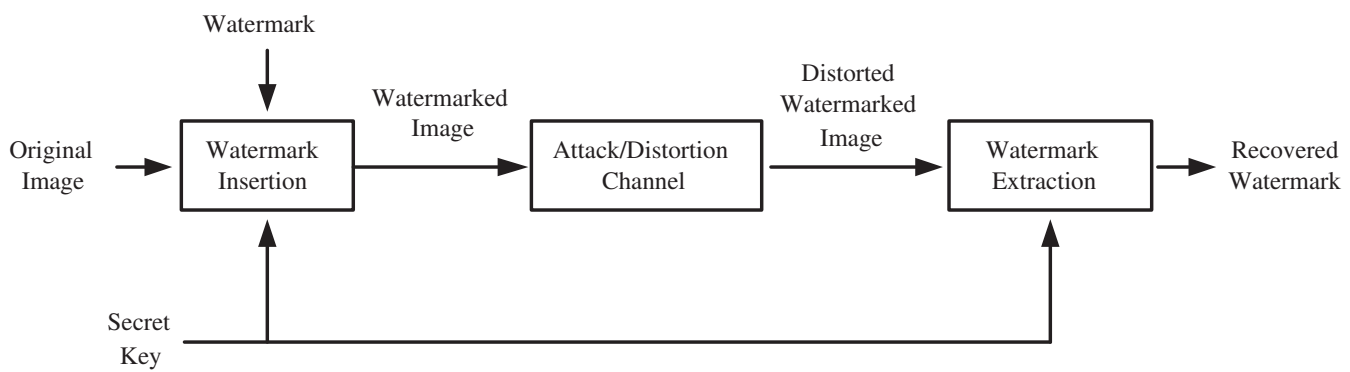

Fig. 1. Generic block diagram of a blind watermarking system.

second row which is a linear (slant) function of the column index. The matrices are formed by an iterative construction that exhibits the matrices as products of sparse matrices, which in turn leads to a fast transform algorithm.

The Slant transform matrix of order two is given by

$$
\mathbf{S}_{2}=\frac{1}{\sqrt{2}}\left[\begin{array}{cc}
1 & 1 \\
1 & -1
\end{array}\right]
$$

The Slant matrix of order four is obtained by the operation

$$
\mathbf{S}_{4}=\frac{1}{\sqrt{2}}\left[\begin{array}{cc|cc}
1 & 0 & 1 & 0 \\
a_{4} & b_{4} & -a_{4} & b_{4} \\
\hline 0 & 1 & 0 & -1 \\
-b_{4} & a_{4} & b_{4} & a_{4}
\end{array}\right] \bullet\left[\begin{array}{c|c}
\mathbf{S}_{2} & \mathbf{0} \\
\hline \mathbf{0} & \mathbf{S}_{2}
\end{array}\right]
$$

where $a_{4}$ and $b_{4}$ are scaling constants. The orthonormality conditions lead to:

$$
a_{4}=2 b_{4} \text { and } b_{4}=\frac{1}{\sqrt{5}} .
$$

Equation (4) can be generalized to give the Slant matrix of order $N$ in terms of the Slant matrix of order $N / 2$ by the following recursive relation:

$$
\begin{aligned}
\mathbf{S}_{N}=\frac{1}{\sqrt{2}} & {\left[\begin{array}{cc|c|cc|c}
1 & 0 & \mathbf{0} & 1 & 0 & \mathbf{0} \\
a_{N} & b_{N} & & -a_{N} & b_{N} & \\
\hline \mathbf{0} & & \mathbf{I}_{\frac{N}{2}-2} & \mathbf{0} & \mathbf{I}_{\frac{N}{2}-2} \\
\hline 0 & 1 & \mathbf{0} & 0 & -1 & \mathbf{0} \\
-b_{N} & a_{N} & & b_{N} & a_{N} & \\
\hline \mathbf{0} & & \mathbf{I}_{\frac{N}{2}-2} & \mathbf{0} & -\mathbf{I}_{\frac{N}{2}-2}
\end{array}\right] } \\
& \bullet\left[\begin{array}{c|c|}
\mathbf{S}_{\frac{N}{2}} & \mathbf{0} \\
\hline \mathbf{0} & \mathbf{S}_{\frac{N}{2}}
\end{array}\right] .
\end{aligned}
$$

The matrix $\mathbf{I}_{\frac{N}{2}-2}$ is the identity matrix of dimension $\frac{N}{2}-2$. The constants $a_{N}, b_{N}$, may be computed by the formulas:

$$
a_{2 N}=\left(\frac{3 N^{2}}{4 N^{2}-1}\right)^{1 / 2} \text { and } b_{2 N}=\left(\frac{N^{2}-1}{4 N^{2}-1}\right)^{1 / 2} \text {. }
$$

\section{WATERMARKING IN SLANT TRANSFORM DOMAIN}

A generic block diagram of a blind watermarking system [?] is shown in Figure 1. The original image is not necessary at the watermark extraction stage. This refers to a "blind" watermarking process. A visually recognizable pattern is embedded by modifying the Slant transform coefficients of relevant sub-blocks of the host image. The detailed imageembedded watermark insertion and extraction algorithm are discussed in this section.

Copyright information in the form of a trademark or logo can be created as a pattern for watermarking. In our experiment, a grayscale image of size $64 \times 64$ is used as the watermark. The watermark embedding process is shown in Figure 2. We adapt a similar image-embedded watermark insertion algorithm as in [7], while using the Slant transform domain instead of the Hadamard transform domain. The algorithm can be described as follows.

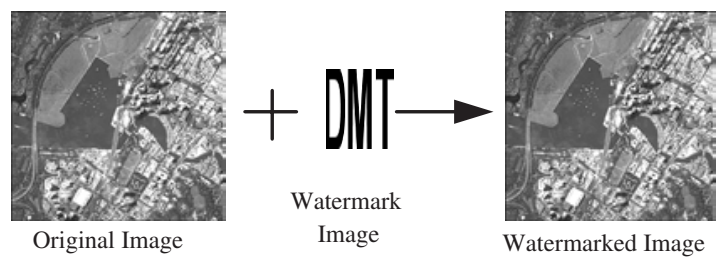

Fig. 2. Image-embedded watermark embedding process.

The watermark image, $\mathbf{W}$, is first transformed into a set of Slant transform coefficients by Equation (1). A Slant transform matrix of order 64 is applied to this image, and then a Slant transform components matrix is obtained. The DC component is stored in the secret key file and the AC components are used for watermark embedding.

Let the original image be $\mathbf{X}$. Similar to the algorithm used in [7, 8], it is decomposed into a set of non-overlapped sub-blocks. An pseudo-random number generator is used to select a certain number of sub-blocks for watermark embedding, whose initial seed is also contained in the secret key file. In every selected sub-block, sixteen middle and high 
frequency coefficients are used for consequent modulation. The coefficient selection affects the performance of the watermarking scheme significantly. The high frequency components are relatively vulnerable to compression operations, while the low frequency components must be retained for visual quality of the watermarked image. Therefore, most existing watermarking schemes choose to embed the watermark into the middle frequency band. In our scheme, embedding locations as shown in Figure 3 are adopted. These locations are selected based on a tradeoff analysis between robustness and data integrity.

Let the watermark Slant transform coefficients denoted by $w_{i}$. The AC coefficients of Slant transformed original image sub-blocks, before and after inserting watermark are denoted by $x_{i}$ and $x_{i}^{*}$, respectively, where $i \in(0, n]$ and $n$ is the number of the watermark coefficients to be embedded into every sub-block, which is set to 16 in our experiment. The embedding formula is given as follows

$$
x_{i}^{*}=\alpha w_{i}
$$

where $\alpha$ is the watermark strength factor that controls the tradeoff between visual quality of the watermarked image and robustness of the watermarking scheme.

After embedding, the original coefficient $x_{i}$ is replaced by $x_{i}^{*}$ and a new $8 \times 8$ matrix of Slant transform components of image sub-block is obtained. The inverse Slant transform is then applied to the matrix using Equation (2) to obtain the luminance matrix of the watermarked image sub-block. After performing the watermark insertion for all the selected sub-blocks of the original image, a watermarked image, $\hat{\mathbf{X}}$, is obtained. At the same time, as indicated earlier, the secret key file has been generated for subsequent decoding.

In watermark detection, the positions of the sub-blocks with watermark embedded are computed using the initial state number of the pseudo-random number generator that is contained in the key file. All the selected sub-blocks are Slant transformed. Let these coefficients denoted by $\hat{x}_{i}^{*}$ and the retrieved watermark Slant transform coefficients by $\hat{w}_{i}$, where $i \in(0, n]$ and $n$ is the number of the watermarked coefficients in every sub-block. The watermark extraction

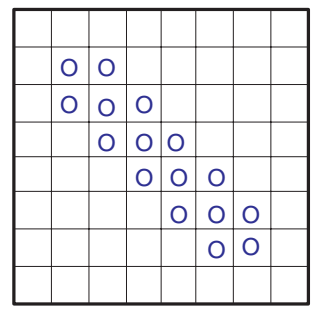

Fig. 3. Embedding locations in one selected Slant transform components block. formula is given by

$$
\hat{w}_{i}=\frac{\hat{x_{i}^{*}}}{\alpha} .
$$

The above extracted AC coefficients and the DC component stored in the key file are rearranged into a Slant transform components matrix. The extracted watermark image, $\hat{\mathbf{W}}$, is then obtained by an inverse Slant transform using Equation (2).

\section{SIMULATION RESULTS}

We use two $512 \times 512$ gray-scale satellite images with different distinct textures to test our algorithm. The original and watermarked images are shown in Figure 4. Results show that there are no perceptually visible degradations on the watermarked images with a PSNR of $37.43 \mathrm{~dB}$ for Singapore and $40.65 \mathrm{~dB}$ for Dolomites.
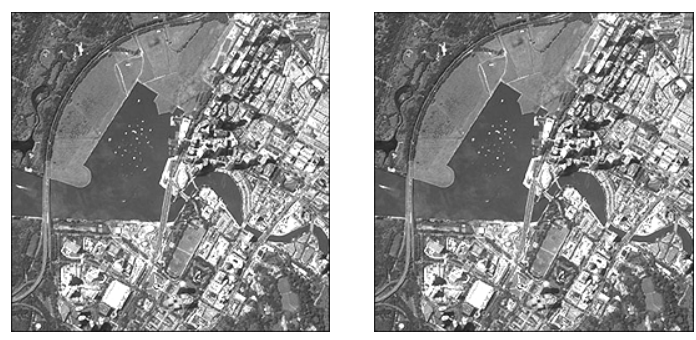

(a)
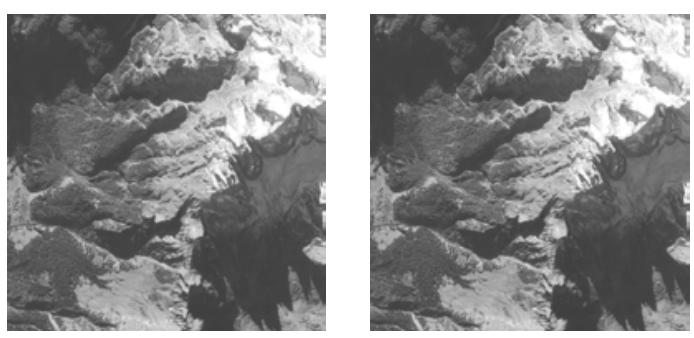

(b)

Fig. 4. Original images (left) and watermarked images (right): (a) Singapore; (b) Dolomites.

The Slant transform watermarking scheme is benchmarked using Stirmark 4 [5] and Checkmark [6]. Sample results of the Singapore are reported in Table 1. We can see that the Slant transform watermarking is robust to additive noise and common image processing techniques.

We also compare the robustness of the Slant scheme to the Hadamard scheme in [7,8] against the typical JPEG compression attack. The results are shown in Figures 5. We can see that, for the two typical textured images, the Slant transform survive JPEG compression far better than the Hadamard transform. Since the Slant transform is designed to match basis vectors to areas of constant lumi- 


\begin{tabular}{|c|c|c|}
\hline $\begin{array}{c}\text { Benchmarking } \\
\text { Operations }\end{array}$ & $\begin{array}{c}\text { Extracted } \\
\text { Watermark }\end{array}$ & $\begin{array}{c}\text { Correlation } \\
\text { Coefficient }\end{array}$ \\
\hline Gaussion filtering & & 0.9640 \\
\hline Wiener filtering & & 0.9500 \\
\hline JPEG compression & & \\
\hline Wavelet compression & & 0.9273 \\
\hline & & \\
\hline Sharpening & & 0.94 \\
\hline Additive noise & & \\
\hline
\end{tabular}

Table 1. Sample benchmarking results for Singapore image.

nance slope, it is good for compact energy in "smooth" images. However, in terms of watermarking, it works better for textured images, for there would be more Slant transform bands remained intact due to the energy spread to more middle or low frequency bands. It follow therefore that the Slant transform is very robust when applied for watermarking textured images, which usually suffer in common image processing operations such as compression.

\section{CONCLUSIONS}

This paper has presented a robust watermarking technique for copyright protection of textured images based on the Slant transform. The embedding and extraction processes have been described in details. The Slant transform has more useful middle and high frequency bands than several high gain transforms, especially for images with complex textures such as satellite images, which provides a good "hidden" space for digital watermarking. This research is based on our previous work on the Hadamard transform [7, 8]. Comparison results between the Slant transform and the Hadamard transform showed the significant advantage of the Slant transform for robust watermarking of complex textured images.

\section{REFERENCES}

[1] R. J. Clarke, Transform Coding of Image, Academic Press, New York, 1985.

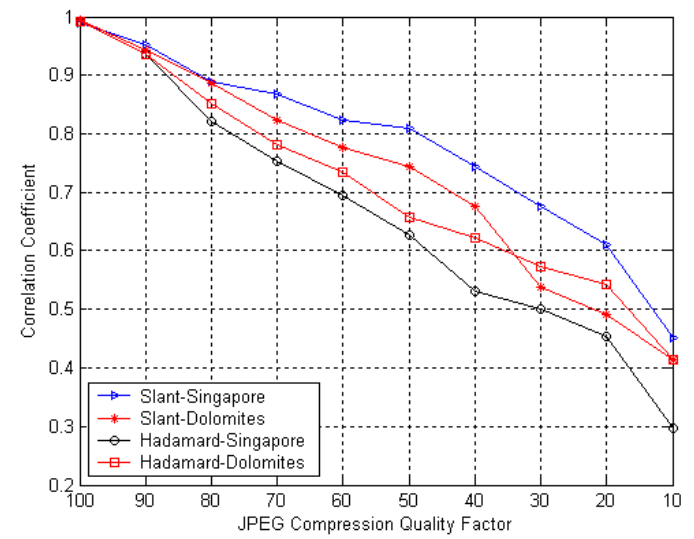

Fig. 5. Performance comparisons between Slant transform and Hadamard transform.

[2] W. K. Pratt, L. R. Welch, and W. H. Chen, "Slant transform for image coding," Proc. Symp. Appl. Walsh Function, Mar. 1972.

[3] W. K. Pratt, W. H. Chen, and L. R. Welch, "Slant transform coding," IEEE Trans. Commun., vol. COM-22, Aug. 1974.

[4] Z. D. Wang, "New algorithm for the slant transform," IEEE Trans. Pattern Anal. Machine Intell., vol. pami-4, pp. 551-555, Dec. 1982.

[5] http://www.cl.cam.ac.uk/ fapp2/watermarking/stirmark/.

[6] http://watermarking.unige.ch/Checkmark/index.html.

[7] A. T. S. Ho, J. Shen, and S. H. Tan, "Digital image-inimage watermarking technique for copyright protection of satellite images using the fast Hadamard transform," in IGARSS'02, Toronto,Canada, June 2002.

[8] A. T. S. Ho, J. Shen, and S. H. Tan, "A robust digital image-in-image watermarking technique for satellite images," in ICICS'01, Singapore, Oct. 2001. 